\title{
Health behaviours and beliefs in individuals with familial pancreatic cancer
}

\author{
Meghan Underhill-Blazey ${ }^{1}$ (D) Traci Blonquist $^{1}$ - Janette Lawrence ${ }^{2} \cdot$ Fangxin Hong $^{1} \cdot$ Matthew B. Yurgelun $^{1,3}$. \\ Sapna Syngal ${ }^{1,3}$
}

Published online: 14 September 2019

(c) The Author(s) 2019

\begin{abstract}
Individuals at high risk for pancreatic cancer are recommended surveillance and healthy lifestyle behaviours and patient experience with recommendations are understudied. To describe engagement and experience with surveillance, tobacco and alcohol use, health beliefs and motivation (Champion Health Belief Measure) and the relationship with personal, psychosocial (Impact of Event Scale), and familial characteristics. Interest in integrative therapies (complementary therapies) are described. A multi-site cross-sectional survey including individuals at high risk for pancreatic cancer with no diagnosis of pancreatic cancer who have been evaluated at a comprehensive cancer center. Descriptive statistics and Wilcoxon rank sum test and Fisher's exact test were used to assess univariate associations. Of the 132 respondents ( $72 \%$ response rate), 92 (70\%) reported undergoing surveillance which was associated with older age $(\mathrm{p}=0.001)$. Of which, $36 \%$ and $51 \%$ report that magnetic resonance imaging (MRI) or endoscopic ultrasound (EUS), respectively, were uncomfortable; $22 \%$ and $30 \%$ dread the next MRI or EUS, respectively. Of those who reported alcohol consumption $(n=88) ; 15 \%$ consumed 1 or more drinks daily and no alcohol consumption was associated with higher Impact of Event scale scores $(p=0.024)$. A total of six participants were currently smoking every day or some days. Participants reported high motivation to engage in heathy behaviours and $92 \%$ were interested in integrative therapies. In these select participants, most were engaging in pancreatic cancer surveillance, alcohol intake was moderate, and tobacco intake was minimal. Modifiable factors, such as experience and comfort with surveillance could be addressed. The sample is motivated to engage in behavioural health intervention.
\end{abstract}

Keywords Pancreatic cancer risk $\cdot$ Surveillance $\cdot$ Health behaviour $\cdot$ Patient reported outcomes

\section{Introduction}

\section{Background/rationale}

Pancreatic cancer is projected to increase from the fourth to the second leading cause of cancer-related deaths by 2030 [1]. Poor outcomes are, in part, due to the few signs and symptoms of pancreatic cancer and the late stage of diagnosis. Only a minority of patients are diagnosed with the

Meghan Underhill-Blazey

meghanl_underhill@dfci.harvard.edu

1 Dana-Farber Cancer Institute, 450 Brookline Avenue, LW 522, Boston, MA 02215, USA

2 Massachusetts General Hospital, 55 Fruit Street, Boston, MA 02114, USA

3 Brigham and Women's Hospital, 75 Francis Street, Boston, MA 02215, USA earliest stage of pancreatic cancer, when surgical cure is most likely [2].

Individuals with risk related to familial or hereditary factors face up to a 3-8-fold increase in risk for developing pancreatic cancer compared to the general population risk of $1.5 \%$, with the highest risk group having known genetic syndromes in which pancreatic cancer is a component [3]. Individuals are most commonly identified as having familial pancreatic cancer risk due to having two or more relatives affected with pancreatic cancer [2,3].

Those at highest risk, or those with a relative risk greater than fivefold and unaffected with cancer, are currently offered cancer surveillance with endoscopic ultrasound (EUS) which requires sedation, and/or magnetic resonance imaging (MRI) annually starting at age 50 or at 10 years prior to the earliest diagnosis of pancreatic cancer in the family [3]. If surveillance identifies a suspicious solid or cystic lesion or intraductal papillary mucinous neoplasm 
(IPMN), the follow-up interval may be shortened or surgical resection may be recommended [3]. Current data suggest that up to $39 \%$ of patients will have an identified abnormality that warrants further evaluation $[4,5]$.

Modifiable behaviours such as current tobacco use, and heavy alcohol consumption are related to a significantly increased relative risk for pancreatic cancer. Therefore, in addition to engaging in surveillance, at-risk individuals are encouraged to maintain healthy lifestyle behaviours, including minimal alcohol intake and avoidance of tobacco [3]. Modifying health behaviours could reduce risk for pancreatic cancer, and also reduce risk for other co-morbid conditions, such as heart disease or diabetes mellitus, or cancers, such as breast cancer, colon cancer, and lung cancer, a cascade benefit of lifestyle modification for pancreatic cancer risk [6].

Individuals living with inherited pancreatic risk may face cancer related worry or psychosocial distress associated with risk and family experience [7, 8]. Integrative therapies (also known as complementary therapies), such as meditation, yoga, or exercise, have been found to reduce feelings of distress and contribute to overall well-being, and are recommended for the care of individuals with cancer [9]. There are limited data related to the perceptions of at risk individuals on integrative therapies, however, these therapies are promising for providing supportive care and further exploration is warranted.

\section{Objectives}

To describe engagement in surveillance, experiences with surveillance, tobacco use, alcohol use, health beliefs and health motivation and the relationship with personal, psychosocial, and familial characteristics. Engagement in and interest in integrative therapies are also described. The analysis presented here is part of a larger study aimed at describing relationships between personal and demographic factors and psychosocial and health behaviour outcomes in individuals with high pancreatic cancer risk. Personal characteristics associated with psychosocial outcomes have been previously reported [7].

\section{Methods}

\section{Study design}

Institutional Review Board approval was obtained from the lead study site. We conducted a multi-site cross-sectional descriptive survey study using a tailored mailed survey method [10]. Surveys were available for completion in paper or online.

\section{Study participants and setting}

Participants were identified from two academic medical centers in the northeastern United States. Eligible participants were English speaking adults with no history of pancreatic cancer who met criteria for inherited or familial pancreatic cancer risk [3] and had been recommended by a physician to undergo pancreatic cancer surveillance. Participants were first sent a mailed information sheet along with the survey, with information pertaining to consent and how to complete the survey either by paper or online. Participants were contacted by mail two more times for recruitment. A $\$ 5$ incentive was provided to all participants in the first mailing.

\section{Variables and measurement}

Preliminary qualitative research conducted by the study team [8] and the Health Belief Model [11] guided the identification of survey items. All measures were self-reported by the participant.

\section{Demographics}

Information pertaining to self-reported personal cancer history, age, marital status, experience caring for a person with cancer (yes/no), experience with familial cancer death (yes/ no) and length of time since that experience (less than or equal 5 years or 6 or more years ago) was collected.

\section{Surveillance and health behaviours}

Participants were asked to report pancreatic cancer surveillance behaviours (endoscopic ultrasound and MRI) or behavioural intentions, and experiences with surveillance (level of comfort with procedure and amount of dread for next procedure [12]). Smoking and alcohol use were measured based on adapted measures from the Health Information National Trends Survey (HINTS) and evaluated based on current American Cancer Society guidelines which recommend tobacco cessation or avoidance and no more than 1-2 alcoholic drinks per day [6]. Use or interest in using other health therapies, or integrative therapies, were assessed with 2 study specific items asking participants to select (1) therapies that they had previously used and (2) therapies that they would consider using in the future. Participants could select all that apply from a list of 17 response options. Options included, for example, massage, acupuncture, meditation, yoga, or nutrition consultation.

Health Belief Model variables were measured using the widely used and validated Champion Health Beliefs scale (CHBS) [13], adapted for pancreatic cancer surveillance 
with permission from the author. The CHBS measures key constructs that predict an action, such as a health behaviour. As posited by the model, susceptibility (e.g. Extremely likely I will get pancreatic cancer in the future) and severity (e.g. the thought of pancreatic cancer scares me) indicate that the participant has recognized a threat, in this case pancreatic cancer, which is necessary to promote health behaviour. Benefits (e.g. when I get pancreatic cancer surveillance I don't worry as much about pancreatic cancer) and barriers (e.g. having pancreatic cancer surveillance would be painful) are specific to the health behaviour, in this example pancreatic surveillance, and to engage a participant must find high benefit and low barriers to the behaviour. Health motivation (e.g. I exercise at least three times a week) is the individual's readiness to be concerned about health promotion and is a determinant of engaging in future health behaviours.

Subscales were scored on a range of 1 (strongly disagree) to 5 (strongly agree) with a higher score indicating more of the construct. If 1 item in the subscale was missing, it was replaced by the average of the remaining items. The possible scores for the susceptibility subscale are from 5 to 25 , for severity $7-35$, and for health motivation 7-35. The benefits subscale (four items, possible scores 4-20) is only asked to those that answered "yes" to engaging in surveillance and the barriers subscale (five items, possible scores 5-25) is only asked to those that answered "no".

Psychosocial factors: Pancreatic cancer risk related distress was measured by The Revised Impact of Event Scale (IES) [14], a 22 item measure validated and widely used to measure the outcome of distress associated with hereditary cancer risk and cancer screening. The measure is scored ranging from 0 to 88 , with higher score indicating more distress and a score of 24 or greater indicating post-traumatic stress. Cancer Risk Perception was measured using adapted version of items included in the Levy et al. publication measuring perceptions of chance of developing pancreatic cancer and how this risk compares to the average person of the same age [15]. For this analysis, we categorized risk perception into $40 \%$ or less or $50 \%$ or greater due to current objective risk estimates for pancreatic cancer which indicate that most objective risk for cancer falls below $40 \%$ [3].

\section{Study size and analysis}

The study was exploratory and therefore no formal power calculation was conducted. All continuous items and measures were summarized with descriptive statistics (median/ range) and compared between groups with the Wilcoxon rank sum test. Categorical items and measured were compared between groups with the Fisher's exact test. Exact 95\% confidence intervals were calculated for screening intent and health behaviours. Due to a small number of participants reported as current smokers, only descriptive data is presented pertaining to smoking.

\section{Results}

\section{Participant characteristics}

Of the 186 individuals sent a survey, 133 responded (72\% response rate) and 132 had analyzable data. Complete demographics and psychosocial characteristics of the study sample are reported elsewhere [7]. All participants were adults who did not have a pancreatic cancer diagnosis. Participants had been evaluated in a comprehensive cancer center by a genetic counselor and physician and have been identified as having high risk for pancreatic cancer due to personal or familial factors. In total, 92 (70\%) indicated having engaged in surveillance. Personal characteristics of participants in relation to engagement in pancreatic cancer surveillance, of the 128 reporting, are presented in Table 1.

\section{Main results}

Surveillance behaviours: Of the 92 participants responding "yes" to having surveillance, 91 reported the type of surveillance. Overall, 16 participants (18\%) reported undergoing EUS alone, 16 (18\%) MRI alone, 57 (63\%) both EUS and MRI, and $2(2 \%)$ reported another type of surveillance. Of the 57 that indicated having both MRI and EUS, 55 answered the item about preference; 27 (49\%) preferred MRI, 5 (9\%) preferred EUS and 20 (36\%) did not have a preference ( 3 responded "other"). For the 92 participants who underwent pancreatic cancer surveillance, 89 answered the question pertaining to a willingness to continue surveillance in the future, and 79 (89\%; 95\% CI 80-94\%) intend to continue. There were no statistically significant demographic factors associated with intent to continue. Table 2 summarizes participants' experiences and level of comfort with undergoing the procedures. In summary, $36 \%$ and $51 \%$ report that MRI or EUS were a little to very uncomfortable, and $21 \%$ and $30 \%$ have some or a lot of dread for the next MRI or EUS procedure, respectively.

Alcohol and tobacco use: In total, 130 participants answered the question regarding alcohol consumption. Of those, 42 (32\%) reported not drinking any alcoholic drinks per week. Nineteen individuals (15\%) reported consuming 7 or more alcoholic drinks per week, 6 of whom (5\%) reported 10 or more a week. All others $(n=69)$ reported consuming between 1 and 6 alcoholic beverages weekly. The proportion of patients that did not drink any alcohol was significantly higher for those with an Impact of Event Scale score indicating post-traumatic stress (greater than or equal to $24, n=17$ ) 
Table 1 Engagement in surveillance based on personal characteristics

\begin{tabular}{|c|c|c|c|c|c|c|c|}
\hline & \multirow{2}{*}{\multicolumn{2}{|c|}{ Overall }} & \multicolumn{4}{|c|}{$\begin{array}{l}\text { Have you ever had pancreatic } \\
\text { cancer surveillance? }\end{array}$} & \multirow[t]{3}{*}{$\overline{p-\text { value }^{\mathrm{b}}}$} \\
\hline & & & \multicolumn{2}{|l|}{ No } & \multicolumn{2}{|c|}{ Yes } & \\
\hline & $\mathrm{N}$ & $\%$ & $\mathrm{~N}$ & $\%$ & $\mathrm{~N}$ & $\%$ & \\
\hline $\mathrm{N}$ & 128 & - & 36 & - & 92 & - & - \\
\hline Gender $^{\mathrm{a}}$ & & & & & & & 0.14 \\
\hline Male & 41 & 32 & 8 & 22 & 33 & 36 & \\
\hline Female & 86 & 67 & 28 & 77 & 58 & 63 & \\
\hline Marital status $^{\mathrm{a}}$ & & & & & & & 0.13 \\
\hline Unmarried/partnered & 36 & 28 & 14 & 39 & 22 & 24 & \\
\hline Married/partnered & 91 & 71 & 22 & 61 & 69 & 75 & \\
\hline Education level $^{\mathrm{a}}$ & & & & & & & 0.73 \\
\hline$\leq$ High school & 10 & 8 & 2 & 6 & 8 & 9 & \\
\hline$>$ High school & 117 & 91 & 34 & 94 & 83 & 90 & \\
\hline Personal history of cancer & & & & & & & 0.44 \\
\hline No & 67 & 52 & 21 & 58 & 46 & 50 & \\
\hline Yes & 61 & 48 & 15 & 42 & 46 & 50 & \\
\hline $\begin{array}{l}\text { Have you taken care of a very ill parent/ } \\
\text { family member }\end{array}$ & & & & & & & 1.00 \\
\hline No & 43 & 34 & 12 & 33 & 31 & 34 & \\
\hline Yes & 85 & 66 & 24 & 67 & 61 & 66 & \\
\hline Have you lost a family member to cancer & & & & & & & 0.097 \\
\hline No & 7 & 5 & 4 & 11 & 3 & 3 & \\
\hline Yes & 121 & 95 & 32 & 89 & 89 & 97 & \\
\hline Have you been recommended screening & & & & & & & $<0.001$ \\
\hline No & 20 & 16 & 15 & 42 & 5 & 5 & \\
\hline Yes & 107 & 84 & 20 & 56 & 87 & 95 & \\
\hline Don’t know & 1 & $<1$ & 1 & 3 & 0 & 0 & \\
\hline Risk perception $^{\mathrm{a}}$ & & & & & & & 0.22 \\
\hline$<50 \%$ & 50 & 39 & 18 & 50 & 32 & 35 & \\
\hline$\geq 50 \%$ & 69 & 54 & 17 & 47 & 52 & 56 & \\
\hline $\mathrm{IES}^{\mathrm{a}}$ & & & & & & & 0.23 \\
\hline IES $<24$ & 106 & 83 & 28 & 78 & 78 & 85 & \\
\hline IES $\geq 24$, indicating PTSD & 16 & 13 & 7 & 19 & 9 & 10 & \\
\hline
\end{tabular}

${ }^{\mathrm{a}} 1$ unreported for gender, marital status, and education; 9 for risk perception; 6 for IES

${ }^{\mathrm{b}} \mathrm{p}$-value excludes unreported compared to those with a score less than 24 (59\% vs. 29\%; $\mathrm{p}=0.024)$.

Out of the total 132 participants, 40 (30\%) indicated smoking 100 or more cigarettes in a lifetime. Of the 40, 39 answered the follow-up question "How often do you now smoke cigarettes?" and $33(85 \%)$ do not smoke cigarettes at all, $3(8 \%)$ smoke some days, and $3(8 \%)$ smoke every day. Of the six participants currently smoking, five are seriously considering quitting in the next 6 months, the remaining one participant did not respond.

Use of other health therapies: 129 participants answered the question regarding use of other health therapies (i.e. integrative therapies) in the past of which 93 (72\%) indicated using some form of integrative therapy (for example, massage, acupuncture, medication). For the item addressing future interest in integrative therapies, 117 participants answered the question of which 108 (92\%; 95\% CI 86-94) indicated being open to using at least one therapy in the future.

Health beliefs and motivation: Fig. 1 describes the score outcomes for the susceptibility and severity, or perceived threat of pancreatic cancer, and general health motivation subscale scores. The distribution of responses within the susceptibility subscale were significantly different based on risk perception $(p<0.001)$ and IES Scores $(p=0.026)$, with those with higher risk perception and higher pancreatic cancer risk related distress perceived more susceptibility. Seriousness subscales scores differed based on gender $(p=0.014)$ and 
Table 2 Self-reported experience with endoscopic ultrasound (EUS) and magnetic resonance imaging (MRI)

\begin{tabular}{lccccc}
\hline & \multicolumn{2}{l}{ Overall } & & \\
\cline { 2 - 3 } & \multicolumn{2}{l}{ MRI $(\mathrm{n}=72)$} & & \multicolumn{2}{l}{ EUS (n=73) } \\
\cline { 2 - 3 } \cline { 5 - 6 } & $\mathrm{N}$ & $\%$ & & $\mathrm{~N}$ & $\%$ \\
\hline Experience & 3 & 4 & & 10 & 14 \\
Very uncomfortable & 23 & 32 & & 27 & 37 \\
A little uncomfortable & 16 & 22 & & 15 & 21 \\
Somewhat comfortable & 30 & 42 & & 21 & 29 \\
Very comfortable & & & & \\
Dread next procedure & 36 & 50 & 34 & 47 \\
Not at all & 21 & 29 & 17 & 23 \\
A little & 8 & 11 & 14 & 19 \\
Somewhat & 7 & 10 & 8 & 11 \\
A lot & & & &
\end{tabular}

IES scale scores $(p<0.001)$ with females and those with more distress perceiving more seriousness. Health motivation, or current perceived engagement in health promotion, significantly differed based on gender $(p=0.015)$, education level $(p=0.016)$, and having taken care of an ill parent or family member $(p=0.029)$, with females, those with greater than a high school education, and having had taken care of a family member reporting higher motivation.

\section{Discussion}

In the present study, we describe health behaviours (pancreatic cancer surveillance and experience, tobacco, alcohol), health beliefs and motivation and the relationship with personal, psychosocial, and familial factors, as well as engagement and interest in integrative therapies in individuals at elevated risk of pancreatic cancer. Overall, most participants engaged in surveillance and intended to continue. A subset did find both surveillance procedures uncomfortable, with EUS being perceived as uncomfortable more frequently compared to MRI. A subset of the sample (4.6\%) reported consuming levels of alcohol that may exceed current American Cancer Society recommendations of 1 to 2 alcoholic drinks per day. Additionally, six individuals were current smokers, five of whom were interested in quitting. Overall

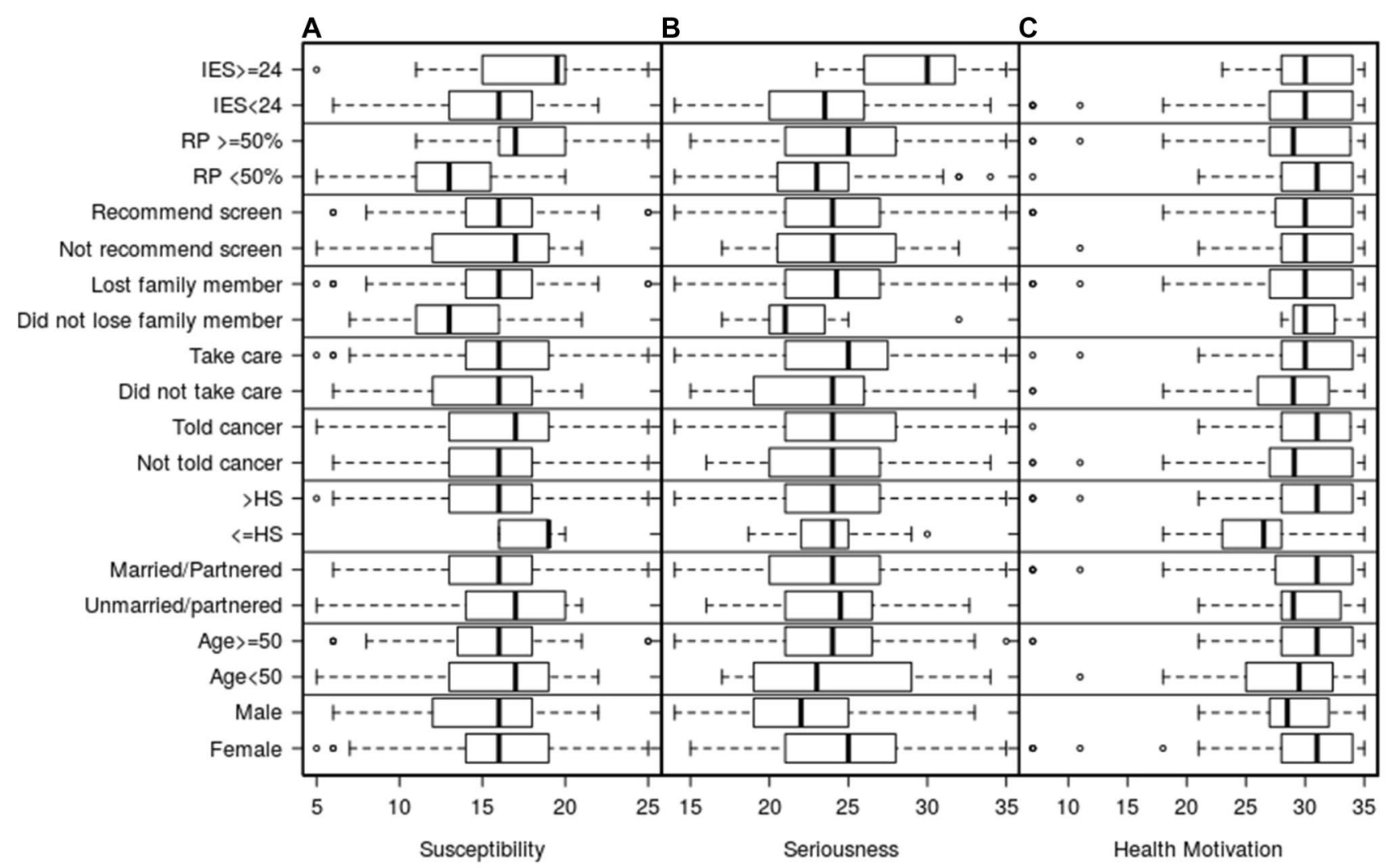

Fig. 1 Distribution of susceptibility, seriousness, and health motivation subscale scores by demographic. Note: IES impact of event scale, $R P$ risk perception; Lost family member/did not lose family member $=$ experience with loss of a family member to pancreatic can- cer; Take care/did not take care $=$ role as a caregiver to a family member with cancer; Told cancer/Not told cancer $=$ personal history of a cancer diagnosis; $\mathrm{HS}=$ high school 
interest or engagement in integrative therapies was high and health motivation was high (ex. engagement in diet and exercise).

Most participants did not find the surveillance MRI uncomfortable and did not dread the next procedure. Of the two surveillance procedures, EUS resulted in more frequent reports of being uncomfortable and slightly more reports of dreading the next procedure. In a longitudinal study measuring the same outcome of comfort after pancreatic surveillance, Konings et al. (2016) had similar findings. Here the authors report that on average $10-11 \%$ of the 140 -person sample felt that MRI or EUS was very uncomfortable respectively and found that $4 \%$ and $11 \%$ dreaded the next procedure at the highest level. Longitudinal findings from the Konings study suggest that after the first EUS, experiences of dread drop to similar levels as found with MRI [12]. In our present study, though some discomfort was reported in this one cross-sectional measurement, most participants intended to continue with surveillance and reported barriers to surveillance are low. These findings suggest participants may benefit from expectation management and support in preparing for the procedure to reduce feelings of discomfort or dread.

Participants reported a moderate perceived susceptibility to pancreatic cancer and that it was a serious disease, therefore indicating that they perceive pancreatic cancer as a threat - a prerequisite to health action. The sample reported high health motivation or readiness to engage, specifically in those with higher education or those who had been a caregiver to an ill family member, with high benefits and few barriers to engaging in pancreatic surveillance. Based on these findings and existing health behaviour theory [16, 17], we find that individuals with high risk for pancreatic cancer may be approachable for health behaviour change intervention.

Additionally, pancreatic cancer risk assessment and consultation, especially for those with heightened risk perception or experience as a caregiver, may be a teachable moment to engage in meaningful behaviour change modification [18]. Our findings further support that participants presenting for pancreatic cancer risk associated care are highly motivated to engage in health behaviour intervention. In part, the reason for this motivation stems from the personal and family experience of living through a loved one's pancreatic cancer diagnosis [8]. Though the sample was a select group engaging in surveillance, recent data suggests that the field of pancreatic cancer genetics is moving towards recommending germline genetic testing in all individuals with pancreatic cancer $[19,20]$. Therefore, soon, there will be a growing number of family members at risk for inherited pancreatic cancer and in whom regular surveillance will have potential health benefits. Therefore, the time of genetic consultation for at risk individuals is an opportune time to promote cancer prevention behaviours and to potentially improve the health of individuals and families impacted by pancreatic cancer.

Previous literature has found that those who seek genetic counseling due to familial cancer risk have similar modifiable cancer risk factors as the general population [21, 22]. In our sample, most individuals were not consuming high levels of alcohol as defined by the American Cancer Society or consuming tobacco. However, a subset report substance use, and current epidemiologic data suggests that heavy alcohol intake of three or more drinks per day and any tobacco exposure can increase risks for developing pancreatic cancer. Tobacco use is particularly concerning and has been noted to lead to a fourfold increase in pancreatic cancer risk in individuals with familial pancreatic cancer and can lead to cancers occurring at a younger age $[23,24]$.

Data suggest that those presenting for genetic consultation or for high risk cancer care are engaging in modifiable behaviours that may contribute to cancer risk. However, despite this knowledge, consultations with genetic teams infrequently discuss modifiable lifestyle factors [25] and are inadequately addressing health promotion related needs. There is a need to further evaluate how to help patients undergoing genetic counseling and testing engage in healthy behaviours. Improving lifestyle and health behaviours in at risk individuals has the cascade benefit of not only reducing a potential for cancer risk but improving overall health and quality of life. Considered in the context that this sample was highly motivated to engage in healthy behaviours, targeted and tailored health messages, that address tobacco or alcohol as necessary, could be an important future clinical and research consideration.

Our findings suggest that not only is risk and risk perception a catalyst for engaging in health promoting activities, experiences within the family are as well and caring for a seriously ill loved one in the context of inherited pancreatic cancer risk is associated with higher reported health motivation. We previously identified that these factors were also associated with higher psychosocial distress compared to others in the same sample [7]. Therefore, when encountering an individual who is at risk for a disease in which they witnessed firsthand as a caregiver, there is an opportunity to promote physical and psychosocial health and wellness.

\section{Limitations}

Study findings are limited to the perspective of participants engaging in care in an academic medical center who are largely female, well-educated and choose to engage in surveillance. Future work could target the enrollment of men to seek a more balanced sample. Additionally, outcome data were self-reported and therefore a potential risk for biased 
responses or socially desirable responses. Finally, we did not collect data pertaining to specific genetic mutation status, Body Mass Index (BMI), diet, or level of physical activity directly as part of this study, which would be important for future studies.

\section{Conclusion}

In these select participants from a comprehensive cancer center, most were engaging in pancreatic cancer surveillance, and alcohol intake was moderate and tobacco intake was minimal. Modifiable factors, such as pancreatic cancer risk related distress and procedural discomfort could be intervened upon within a clinical or research context. The sample is highly motivated to engage in behavioural health intervention. It is imperative that clinical research in the era of precision medicine continues to mandate that effort be made to help individuals prevent cancer by improving health behaviours in themselves or their family. Focusing on improving overall health and wellness through healthy lifestyle behaviours (such as tobacco cessation or reduction of alcohol) is non-invasive, often inexpensive and easily accessible way to holistically promote health and potentially reduce illness in an already vulnerable population of individuals living with high risk for pancreatic cancer.

Acknowledgements We acknowledge Sloane Furniss, PhD, and her feedback and review of the final manuscript.

Funding Funding provided by DFCI Mittleman Family Fund, KL2/ Catalyst Medical Research Investigator Training award (National Center for Research Resources and the National Center for Advancing Translational Sciences, National Institutes of Health Award KL2 TR001100). Additional funding for SS by U01 CA210170 (PI Goggins) and the DFCI Bowen-Chapman Family Research Fund for Pancreatic Cancer Prevention.

\section{Compliance with ethical standards}

Conflict of interest Dr. Syngal reports being a consultant for Myriad Genetics, Inc. and Digital China Health, Inc.

Open Access This article is distributed under the terms of the Creative Commons Attribution 4.0 International License (http://creativeco mmons.org/licenses/by/4.0/), which permits unrestricted use, distribution, and reproduction in any medium, provided you give appropriate credit to the original author(s) and the source, provide a link to the Creative Commons license, and indicate if changes were made.

\section{References}

1. Rahib L, Smith BD, Aizenberg R, Rosenzweig AB, Fleshman JM, Matrisian LM (2014) Projecting cancer incidence and deaths to 2030: the unexpected burden of thyroid, liver, and pancreas cancers in the United States. Can Res 74(11):2913-2921. https:// doi.org/10.1158/0008-5472.can-14-0155

2. American Cancer Society (ACS) (2011) Pancreatic Cancer http:// www.cancer.org/acs/groups/cid/documents/webcontent/00313 1-pdf.pdf. Accessed 1 Aug 2011

3. Syngal S, Brand RE, Church JM, Giardiello FM, Hampel HL, Burt RW (2015) ACG clinical guideline: genetic testing and management of hereditary gastrointestinal cancer syndromes. Am J Gastroenterol 110(2):223. https://doi.org/10.1038/ ajg.2014.435

4. Canto MI, Hruban RH, Fishman EK, Kamel IR, Schulick R, Zhang Z, Topazian M, Takahashi N, Fletcher J, Petersen G (2012) Frequent detection of pancreatic lesions in asymptomatic highrisk individuals. Gastroenterology 142(4):796-804. https://doi. org/10.1053/j.gastro.2012.01.005

5. Harinck F, Nagtegaal T, Kluijt I, Aalfs C, Smets E, Poley J-W, Wagner A, Van Hooft J, Fockens P, Bruno M (2011) Feasibility of a pancreatic cancer surveillance program from a psychological point of view. Genet Med 13(12):1015-1024. https://doi. org/10.1097/GIM.0b013e31822934f5

6. Kushi LH, Doyle C, McCullough M, Rock CL, Demark-Wahnefried W, Bandera EV, Gapstur S, Patel AV, Andrews K, Gansler T (2012) American Cancer Society Guidelines on nutrition and physical activity for cancer prevention: reducing the risk of cancer with healthy food choices and physical activity. CA: Cancer J Clin 62(1):30-67. https://doi.org/10.3322/caac.20140

7. Underhill M, Hong F, Lawrence J, Blonquist T, Syngal S (2018) Relationship between individual and family characteristics and psychosocial factors in persons with familial pancreatic cancer. Psycho-Oncology 27(7):1711-1718. https://doi.org/10.1002/ pon. 4712

8. Underhill M, Berry D, Dalton E, Schienda J, Syngal S (2015) Patient experiences living with pancreatic cancer risk. Hered Cancer Clin Pract 13(1):13. https://doi.org/10.1186/s1305 3-015-0034-1

9. Greenlee H, DuPont-Reyes MJ, Balneaves LG, Carlson LE, Cohen MR, Deng G, Johnson JA, Mumber M, Seely D, Zick SM, Boyce LM, Tripathy D (2017) Clinical practice guidelines on the evidence-based use of integrative therapies during and after breast cancer treatment. CA: Cancer J Clin 67(3):194-232. https://doi. org/10.3322/caac. 21397

10. Dillman DA, Smyth JD, Christian LM (2014) Internet, phone, mail, and mixed-mode surveys: the tailored design method. John Wiley \& Sons, Hoboken

11. Skinner CS, Tiro J, Champion VL (2015) The health belief model. In: Glanz K, Rimer BK, Viswanath K (eds) Health behavior: theory, research, and practice, 5th edn. Jossey-Bass, San Francisco, pp 75-94

12. Konings IC, Sidharta GN, Harinck F, Aalfs CM, Poley JW, Kieffer JM, Kuenen MA, Smets E, Wagner A, Hooft JE (2016) Repeated participation in pancreatic cancer surveillance by high-risk individuals imposes low psychological burden. Psycho-Oncology 25(8):971-978. https://doi.org/10.1002/pon.4047

13. Champion VL (1999) Revised susceptibility, benefits, and barriers scale for mammography screening. Res Nurs Health 22(4):341-348

14. Horowitz M, Wilner N, Alvarez W (1979) Impact of Event Scale: a measure of subjective stress. Psychosom Med 41(3):209-218

15. Levy AG, Shea J, Williams SV, Quistberg A, Armstrong K (2006) Measuring perceptions of breast cancer risk. Cancer Epidemiol Prev Biomark 15(10):1893-1898

16. Rosenstock IM (1974) Historical origins of the health belief model. Health Educ Monogr 2(4):328-335 
17. Glanz K, Rimer BK, Viswanath K (2008) Health behavior and health education: theory, research, and practice. John Wiley \& Sons, Hoboken

18. McBride CM, Puleo E, Pollak KI, Clipp EC, Woolford S, Emmons KM (2008) Understanding the role of cancer worry in creating a "teachable moment" for multiple risk factor reduction. Soc Sci Med 66(3):790-800

19. Syngal S, Furniss C (2018) Germline genetic testing for pancreatic ductal adenocarcinoma at time of diagnosis. JAMA 319(23):2383-2385. https://doi.org/10.1001/jama.2018.6227

20. Hu C, Hart SN, Polley EC et al (2018) Association between inherited germline mutations in cancer predisposition genes and risk of pancreatic cancer. JAMA 319(23):2401-2409. https://doi. org/10.1001/jama.2018.6228

21. Quillin JM (2016) Lifestyle risk factors among people who have had cancer genetic testing. J Genet Couns 25(5):957-964. https ://doi.org/10.1007/s10897-015-9925-6

22. Burton AM, Peterson SK, Marani SK, Vernon SW, Amos CI, Frazier ML, Lynch PM, Gritz ER (2010) Health and lifestyle behaviors among persons at risk of Lynch syndrome. Cancer
Causes Control 21(4):513-521. https://doi.org/10.1007/s1055 2-009-9482-0

23. Midha S, Chawla S, Garg PK (2016) Modifiable and non-modifiable risk factors for pancreatic cancer: a review. Cancer Lett 381(1):269-277. https://doi.org/10.1016/j.canlet.2016.07.022

24. Becker AE, Hernandez YG, Frucht H, Lucas AL (2014) Pancreatic ductal adenocarcinoma: risk factors, screening, and early detection. World J Gastroenterol: WJG 20(32):11182-11198. https:// doi.org/10.3748/wjg.v20.i32.11182

25. Albada A, Vernooij M, van Osch L, Pijpe A, van Dulmen S, Ausems MG (2014) Does and should breast cancer genetic counselling include lifestyle advice? Fam Cancer 13(1):35-44. https:// doi.org/10.1007/s10689-013-672-5

Publisher's Note Springer Nature remains neutral with regard to jurisdictional claims in published maps and institutional affiliations. 